\title{
Tear Film Evaluation in Contact Lens Wearers and Non Wearers
}

\author{
Shrestha G.S. , Sujakhu D. , Shrestha J. B., Shrestha J. K. \\ B.P. Koirala Lions Centre for Ophthalmology Studies, Institute of Medicine, Tribhuvan University, Mechi Eye Hospital, \\ Jhapa, Nepal \\ Correspondence address: Gauri Shankar Shrestha, B.P. Koirala Lions Centre for Ophthalmic Studies, Maharajgunj, \\ Kathamndu, P. O. Box no 8750
}

E-mail: gs101lg@hotmail.com

\begin{abstract}
Background: Dry eye symptoms are more frequent in contact lens wearers than in non wearers. Dry eye is considered relatively common in contact lens wearers than non-wearers. However, there was no any report in our clinical setting that compared and addressed the issue related to dry eye and its symptom in contact lens wearers. The present study was conducted to compare pre-ocular tear film in contact lens wearers (CLW) and contact lens non wearers (CLNW), and evaluate the relationship between clinical and subjective assessment of dry eye symptoms.
\end{abstract}

Methods: It was a comparative study conducted among 131 subjects (262 eyes) attended in the contact lens clinic at BP Koirala Lions Center for the Ophthalmic Studies. Sixty five were daily soft contact lens wearers; sixty-six were non wearers.

Tear function assessment included non- invasive tear break up time, invasive tear break up time, tear prism height and Schirmer II test. Subjective assessment of dry eye symptoms was carried out using McMonnies questionnaires.

Results: Mean age of CLNW was 23.01 \pm 5.53 and CLW was 23.6 \pm 4.61 . Female (89 subjects) visited more than male (42 subjects) in contact lens clinic. Mean symptom score in CLW was significantly higher than CLNW $(\mathrm{p}<0.001)$. But, distribution of symptomatology was not significantly different in both groups. Symptomatic subjects were elder $(p<0.001)$ and female $(\mathrm{p}<0.05)$ in CLNW and only elder subjects in CLW. Tear function tests were significantly lower in symptomatic subjects in CLW and CLNW; Overall tear function test scores were also lower in CLW than CLNW. Tear function tests scores were insignificantly different in male and female. Coefficient of correlation was positive and weak for all tear function tests scores.

Conclusions: Though, tear function tests scores were reduced in CLW than CLNW, dry eye symptomatology was almost similar. Subjective assessment of symptoms is as important as clinical tests.

Key words: tear functions, symptoms, dry eye

\section{Introduction}

Tear film (TF) is a highly specialized and carefully ordered fluid structure which forms an integral part of the ocular surface. Abnormalities in the consistency or volume cause dysfunction of the ocular surface and can ultimately affect the transparency of the cornea. 1
An adequate and stable tear film is necessary to sustain CL wear. Otherwise, several changes occur to TF and adnexa like thinning of TF, lipid layer disruption, increase in mucus secretion, changes in blink characteristics, lid conformity. $2-4$

Dry eye is prevalent in CL wear with $20-30 \%$ of CL wearers 
having dry eye symptoms.5 It was hypothesized that CL wear leads to changes in the structure or the production of the meibomian glands leading to alterations in lipid layer thickness, TF instability, an increased tear osmolarity and dehydration of hydrogel lenses. 6

Recent studies have demonstrated that questioning of SCL wearers about symptoms has more diagnostic value than clinical testing.7 McMonnies developed a dry eye questionnaire8 and found a high level of sensitivity in detecting both dry eye and marginal dry eye.

The purpose of the study was to compare pre-ocular tear film in contact lens wearers (CLW) and contact lens non wearers (CLNW), and evaluate the relationship between clinical and subjective assessment of dry eye symptoms.

\section{Methods}

Subjects and study design

In a hospital based cross sectional and comparative study, 65 daily soft contact lens wearers; 66 non wearers 131 subjects were recruited from the contact lens clinic, BP Koirala Lions Center for the Ophthalmic Studies, Institute of Medicine, Tribhuvan University from November 2006 to April 2007. Myopic subjects having contact lens wear for minimum of 10 hours/day for more than six months and having no obvious ocular pathology on slit lamp examination, were selected as a contact lens wearer (CLW). Age matched myopic subjects, having no history of contact lens use prior, and having no obvious ocular pathology, were selected as contact lens non wearers (CLNW). Presbyopic subjects and systemic medicine users were excluded from the study.

The purpose and procedure of study were clearly explained to and verbal consent was received from all subjects. All the relevant clinical findings tear film evaluation, and subjective responses were recorded. Detailed slit lamp examination was done in a consistent, orderly fashion from eyelid margin, lashes, puncta, and palpebral and bulbar conjunctiva including fornices and cornea.

All collected data were recorded in proforma and entered in computer database for statistical analysis. The Microsoft Excel 2007 program was used for this purpose. The t-test was performed for tear function scores CLW and CLNW. Chi square test was performed for symptomatology in the association of gender and age group. Pearson correlation coefficients of tear function scores were performed to analyze the significance. Confidential interval was set at $95 \%$. P value was considered significant for score equal to or less than 0.05
Assessments

Non- invasive tear break up time (NIBUT): NIBUT was assessed as the time taken for the keratometer mires images to become distorted or out of focus after a complete blink while the eye remained open. Three consecutive readings were noted and the average of these three was considered for the evaluation. A cut off value of less than 10 seconds was considered abnormal.9,10

Invasive tear break up time (IBUT): IBUT was assessed as the time taken for the first dry patch to appear in cornea after instilling fluorescein dye, examining in slit lamp under cobalt blue filter, and making a subject complete blink while the eye remained open. A cut off point was designated as 10 seconds. 11.12

Tear prism height (TPH): Lower lid tear prism height was measured after instilling fluorescein dye under cobalt blue filter in slit lamp graticule and $16 \times$ magnifications. A meniscus height of $<0.2 \mathrm{~mm}$ was considered abnormal whereas between 0.2 and $0.3 \mathrm{~mm}$ was normal.13

Schirmer II test (with anesthesia): A pre-calibrated 35mm× $5 \mathrm{~mm}$ size filter paper strips (Whatman no. 41) were used to measure the amount of tears that was produced over a period of 5 minutes. Before the test, a drop of $4 \%$ lidocaine hydrochloride solution was instilled into the lower cul- desac in each eye. The subjects were instructed to close eyes for 5 minutes to reduce the irritation. Overflowing tear was soaked and makes the surface dry to avoid the false error before the measurement. Reference wetting value was considered with $\mathrm{mm}$ scale imprinted. A cut off value was assigned as $10 \mathrm{~mm}$ in 5 minutes.

Subjective assessment: McMonnies dry eye questionnaire8 was used in each subject (lens wearers and non wearers) to subdivide the subjects into symptomatic and asymptomatic. There were altogether 12 questions. A score of 20 was an indicator of dry eye.

\section{Results}

Distribution of subjects and symptomatology

A total of 131 myopic subjects (262 eyes) were evaluated (table 1) age ranged between 15 to 37 years. They were divided in to two groups contact lens non-wearers (66 subjects), and contact lens wearers (65 subjects). Female significantly enrolled more than male in both groups in the study. 
Table 1: Age, gender and symptomatology among subjects in the study

\begin{tabular}{|c|c|c|c|c|c|}
\hline \multicolumn{2}{|l|}{ Particulars } & Overall $(n=131)$ & $\begin{array}{l}\text { Contact lens non } \\
\text { wearers }(n=66)\end{array}$ & $\begin{array}{l}\text { Contact lens } \\
\text { wearers }(n=65)\end{array}$ & $\mathbf{P *}$ \\
\hline \multicolumn{2}{|l|}{ Age (years)\# } & $23.31 \pm 5.09$ & $23.01 \pm 5.53$ & $23.6 \pm 4.61$ & NS \\
\hline \multirow[t]{2}{*}{ Gender** } & Male & $42(32.48 \%)$ & $27(40.90 \%)$ & $15(23.07 \%)$ & 0.05 \\
\hline & Female & $89(67.52 \%)$ & $39(59.10 \%)$ & $50(76.93 \%)$ & \\
\hline \multirow[t]{2}{*}{$\operatorname{Symptom} \oplus$} & Asymptomatic & $105(80.15 \%)$ & $55(83.44 \%)$ & $50(76.93 \%)$ & NS \\
\hline & symptomatic & $26(19.75 \%)$ & $11(16.66 \%)$ & $15(23.07 \%)$ & \\
\hline \multicolumn{6}{|c|}{$p^{*}$ Wearers Vs non wearers, NS $=$ not significant at $p<0.05$} \\
\hline
\end{tabular}

The mean age of the subjects was $23.01 \pm 5.53$ years for contact lens non-wearers (CLNW) and 23.6 \pm 4.61 for contact lens wearers (CLW). Of the $66 \mathrm{CLNW}, 55$ were asymptomatic and 11 were symptomatic; and of the 65 CLW, 50 were asymptomatic and 15 were symptomatic; this distribution was not significant. Mean age of symptomatic CLNW (29.36 \pm 8.33) was significantly higher $(\mathrm{p}=0.00)$ than mean age of asymptomatic CLNW (21.83 \pm 4.06$)$. Likewise (Table 2$)$, Mean age of symptomatic CLW $(25.6 \pm 4.77)$ was significantly higher $(\mathrm{p}=0.00)$ than mean age of asymptomatic CLW $(23 \pm$ 4.44). In CLNW, females were more symptomatic than male ( $\mathrm{p}=0.05)$; but, it was not significant in CLW.

Table 2: Symptomatology in Contact lens non-wearers and contact lens wearers

\begin{tabular}{|c|c|c|c|c|c|c|}
\hline & \multicolumn{2}{|c|}{ Contact lens non wearers (CLNW) } & \multicolumn{3}{|c|}{ Contact lens wearers (CLW) } & \multirow[b]{2}{*}{$\mathrm{P}^{* *}$} \\
\hline & Asymptomatic & Symptomatic & $\mathrm{P} *$ & Asymptomatic & Symptomatic & \\
\hline Age \# & $21.83 \pm 4.06$ & $29.36 \pm 8.33$ & 0.00 & $23 \pm 4.44$ & $25.6 \pm 4.77$ & 0.00 \\
\hline Male $\Phi$ & $25(92.60 \%)$ & $2(7.40 \%)$ & 0.05 & $11(73.33 \%)$ & $4(26.66 \%)$ & NS \\
\hline Female $\phi$ & $30(76.92 \%)$ & $9(23.08 \%)$ & & $39(78 \%)$ & $11(22 \%)$ & \\
\hline \multicolumn{7}{|c|}{$\mathrm{NS}=$ not significant at $\mathrm{P}<0.05$} \\
\hline \multicolumn{7}{|c|}{ p* significance between Asymptomatic Vs Symptomatic in CLNW; } \\
\hline \multicolumn{7}{|c|}{$\mathrm{p}^{* *}$ significance between Asymptomatic Vs Symptomatic in CLW } \\
\hline \multicolumn{7}{|c|}{ \# Unpaired t- test was performed for the mean age in both CLNW and CLW } \\
\hline$\$$ Chi-squ & or gender in both & NW and CLW & & & & \\
\hline
\end{tabular}

Tear function Evaluation and symptomatology in CLNW and CLW

Non invasive tear break up time (NIBUT): The mean NIBUT was found significantly higher in asymptomatic CLNW $(\mathrm{p}=0.00)$ and asymptomatic CLW $(\mathrm{p}=0.00)$ than symptomatic CLNW and systematic CLW respectively (Table. 3$)$. NIBUT was also significantly higher in CLNW $(\mathrm{p}=0.00)$ than CLW showing that the asymptomatic subgroup had a more stable tear film than the symptomatic subgroup. There was significant correlation between right eye and left eye $(\mathrm{r}=0.91, \mathrm{p}=0.05)$ for NIBUT. Correlation between NIBUT and symptom scores was positive, but, poor $(r=0.258 ; \mathrm{p}=0.05)$.

Invasive tear break up time (IBUT): The mean IBUT was higher in CLNW than CLW ( $<<0.001)$. The mean IBUT was also found significantly higher in asymptomatic CLNW $(p=0.00)$ and CLW $(p=0.00)$ than symptomatic CLNW and CLW respectively (Table 3). This finding also revealed that the asymptomatic subjects had more stable tear film than 
symptomatic subjects. There was significant correlation between right eye and left eye $(\mathrm{r}=0.91, \mathrm{p}=0.05)$ for IBUT. The correlation between symptom scores and IBUT was positive, but, poor $(\mathrm{r}=0.254 ; \mathrm{p}=0.05)$.

Schirmer II test: The mean tear volume by Schirmer II value (Table 3) was significantly higher in CLNW than CLW $(\mathrm{p}=0.00)$. Similarly, the tear volume was also significantly higher in asymptomatic CLNW and CLW than symptomatic CLNW $(\mathrm{p}<0.001)$ and CLW $(\mathrm{p}=0.00)$ respectively. There was significant correlation between right eye and left eye $(\mathrm{r}=0.82, \mathrm{p}=0.05)$ for Schirmer II. The correlation between symptom scores and Schirmer II was positive, but, poor $(\mathrm{r}=$ $0.207, \mathrm{p}=0.05)$.

Tear prism height (TPH): The mean TPH (Table 3 ) was significantly higher in CLNW than CLW (p $<0.001)$. It was also significantly higher in asymptomatic CLNW $(\mathrm{p}=0.00)$ and $\mathrm{CLW}(\mathrm{p}=0.00)$ than symptomatic CLNW and CLW respectively. There was significant correlation between right eye and left eye $(\mathrm{r}=1.0, \mathrm{p}=0.05)$ for TPH, But, the correlation between symptom scores and TPH was insignificant $(\mathrm{r}=0.006 ; \mathrm{p}=0.05)$.

Table 3: Tear function evaluation in contact lens wearers (CLW) and non wearers (CLNW)

\begin{tabular}{|c|c|c|c|c|c|c|c|c|}
\hline \multirow[t]{2}{*}{ Tear Film } & \multicolumn{4}{|c|}{ Contact lens Non-wearer ( $n=132$ eyes) } & \multicolumn{4}{|c|}{ Contact lens wearers ( $n=130$ eyes) } \\
\hline & Overall & Sym & Asym & $\mathbf{P}^{*}$ & Overall & Sym & Asym & $\mathbf{P}^{* * *}$ \\
\hline $\begin{array}{l}\text { N I B U T } \\
\text { (sec) }\end{array}$ & $\begin{array}{l}18.06 \# \\
\pm 6.22\end{array}$ & $\begin{array}{l}14.72 \\
\pm 6.37\end{array}$ & $\begin{array}{l}17.96 \pm \\
6.21\end{array}$ & $<0.001$ & $\begin{array}{l}17.10 \# \pm \\
6.08\end{array}$ & $\begin{array}{l}15.73 \pm \\
5.19\end{array}$ & $\begin{array}{l}17.52 \pm \\
6.28\end{array}$ & 0.002 \\
\hline IBUT (sec) & $\begin{array}{l}16.40 \varnothing \\
\pm 6.09\end{array}$ & $\begin{array}{l}13.18 \pm \\
6.52\end{array}$ & $\begin{array}{l}16.21 \pm \\
6.06\end{array}$ & $<0.001$ & $\begin{array}{l}15.05 \text { ष } \\
\pm 5.92\end{array}$ & $\begin{array}{l}13.66 \pm \\
5.12\end{array}$ & $\begin{array}{l}15.47 \pm \\
6.10\end{array}$ & 0.0019 \\
\hline $\begin{array}{ll}\mathrm{SCH} & \text { II } \\
(\mathrm{mm}) & \end{array}$ & $\begin{array}{l}24.18 \notin \pm \\
9.56\end{array}$ & $\begin{array}{l}20.18 \pm \\
10.75\end{array}$ & $\begin{array}{l}24.40 \pm \\
9.46\end{array}$ & $<0.001$ & $\begin{array}{l}21.84 \phi \\
\pm 9.38\end{array}$ & $\begin{array}{l}19.06 \pm \\
9.48\end{array}$ & $\begin{array}{l}22.68 \pm \\
9.23\end{array}$ & $<0.001$ \\
\hline TPH (mm) & $\begin{array}{l}0.19 \$ \\
\pm 0.04\end{array}$ & $\begin{array}{l}0.16 \\
\pm 0.06\end{array}$ & $\begin{array}{l}0.19 \pm \\
0.04\end{array}$ & $<0.001$ & $\begin{array}{l}0.17 \notin \pm \\
0.05\end{array}$ & $\begin{array}{l}0.15 \pm \\
0.05\end{array}$ & $\begin{array}{l}0.178 \pm \\
0.05\end{array}$ & $<0.001$ \\
\hline \multicolumn{9}{|c|}{$\begin{array}{l}\text { Sym }=\text { symptomatic; Asym }=\text { asymptomatic } ; \text { NIBUT }=\text { non invasive tear break up } \text { IBUT }=\text { Invasive tear } \\
\text { break up; } \mathrm{SCH}=\text { Schirmer test } \mathrm{TPH}=\text { tear prism height } ; \mathrm{sec}=\text { seconds } \mathrm{mm}=\text { millimeters }\end{array}$} \\
\hline \multicolumn{9}{|c|}{$\mathrm{P} \#=0.006$ overall (wearer vs. non wearers) for NIBUT by unpaired t-test; } \\
\hline $\mathrm{P} \Phi<0.001 \mathrm{o}$ & verall (wearer & rvs. non y & arers) for & BUT; TPI & and SCH II $b$ & by unpaired & t-test & \\
\hline
\end{tabular}

Tear functions: gender distribution

NIBUT, IBUT, and Schirmer II test were significantly reduced $(\mathrm{p}<0.05)$ in both male and female CLW than non wearers; but, TPH was significantly reduced only in female CLW than non wearers (Table 4). Tear function scores were insignificantly different between male and female for both CLNW and CLW. 
Table 4: Gender distribution of tear film tests

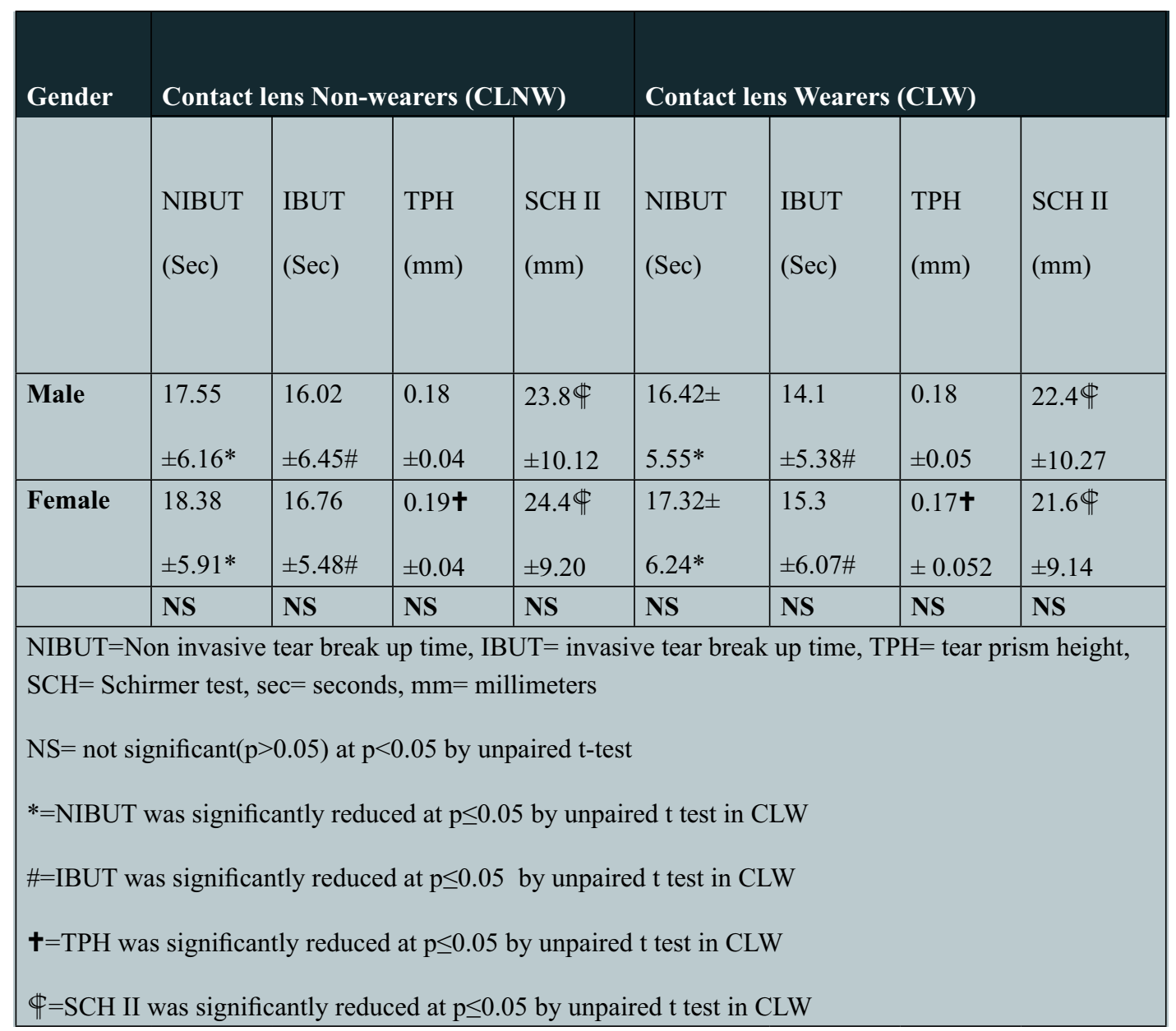

\section{Discussion}

Among 131 subjects, female enrollment was found to be more $(p=0.05)$ than male in both CLW and CLNW. Contact lens dispensing was higher in female than male in our clinical set up. This clinical finding of females' preferences in wearing contact wearing was closely resembled to other recent studies.10,14 The age distribution among CL wearers $(23.6 \pm 4.61 \mathrm{yrs})$ and non wearers (23.01 \pm $5.53 \mathrm{yrs})$ was not significant $(\mathrm{p}=0.49)$. CL wearers in our study were relatively younger than that reported in other literatures;10,14 however similar age range was found in Chopra et al15and Miller et al studies.16 Contact lens dispensing was limited to single vision soft and RGP lenses in our clinics, and Presbyopic contact lens trial and dispensing was not the routine.
Distribution of symptom scores was not significantly different between CLW and CLNW, However, Guillon et al study10 showed significantly high incidence of dry eye symptoms in wearers. Minimum duration of contact lens wear was designated as six months in our study; contact lens wearers were relatively younger; contact lens wearing duration might not be too long in our subjects. Further study on symptomatology of dry eye with duration of contact lens wear is warranted.

In CLNW, symptomatic subjects were relatively older $(\mathrm{p}=0.00)$ and female $(\mathrm{p}=0.05)$ than asymptomatic subjects (Table 2). But, in CLW, symptomatic subjects were only relatively older $(\mathrm{p}=0.00)$. In a study conducted by Glasson et al, 17 gender was found to be strongly associated with dry eye status $(p=0.00)$. In our study, sample size was too small and lacked homogeneity. This could be the cause for 
inconsistency in report for symptomatology in gender.

Mean values of NIBUT $(p=0.006)$, IBUT $(p<0.001)$, TPH $(p<0.001)$ and Schirmer II $(p<0.001)$, measured were greater in non wearers than in wearers (Table 3 ). Toit et al18 showed that there was statistically significant difference between the measurements at the initial visit and final visit after 6 months of contact lens wear $(p=0.01)$. Miller et al16 also reported that there was decrease tear volume (TMH and PRT) in wearers than in non wearers. Similarly, tear function tests (NIBUT, INUT, TPH, and Schirmer II) were reduced in symptomatic subjects than asymptomatic subjects in both CLNW and CLW in our study. Fonn et al19 reported significant reduction of prelens NIBUT in symptomatic wearers. Glasson et al27also found significant lower NIBUT and tear meniscus area in symptomatic contact lens wearers. However, Guillon et al10 result didn't show any significant difference in all tear function tests between wearers and non wearers, however, significant difference was reported between symptomatic and asymptomatic subjects. Chopra et al15 reported statistically insignificant TBUT difference between wearers and non wearers. Santodomingo et al20 found no significant changes in NIBUT and TMH $(\mathrm{p}>0.05)$ in wearers and non wearers. There was a greater variability in tear function tests. Correlation between tear functions and symptom scores was poor; the weakest was the TPH with symptomatology (Figure 1). Though, symptom scores were found higher in CLW than CLNW (Table 1), distribution of symptomatology was not significantly different. Reason for poor correlation could be firstly because of normal tear function scores in both CLNW and CLW, though, it was found reduced in CLW than CLNW. Secondly, the symptoms could be directly related to the presence of Contact lens in contact lens wearers.10 This finding suggested that the instrumental as well as observational bias in the measurement were obvious, and more reliable clinical techniques are necessary to find out dry eye in contact lens wearers.

There was significant correlation between the right and left eyes of the same subject for all the tear function tests. Guillon et al10 also reported the tear film stability correlate for two eyes of the same subject $(\mathrm{p}<0.001, \mathrm{r}=$ 0.97). Similarly, a study by Kallarackal et al21 showed strong correlation between RE and LE for the tear tests (ST, TBUT and FMT). There were no significantly different tear function distributions in male and females in our study. This finding was supported by Chopra et al15 for TBUT values in gender.

\section{Conclusion:}

Tear functions and subjective symptoms were important tools to assess dry eye in contact lens wearers. Specially NIBUT and TBUT should be a routine clinical testing tools. Advanced aged contact lens wearers should have had more attention than non wearers.

\section{Acknowledgement}

We would like to express our gratitude to Mr. Prakash Paudel, PhD research fellow for their assistance and support while conducting the study.

\section{References}

1. Lemp MA. Report of National Eye Institute/ Industry workshop on clinical trials in dry eye. CLAO J 1995;21:4:221-32.

2. Albietz JM. Dry eye: an update on clinical diagnosis, management and promising new treatments. Clin Exp Optom 2001;84:4-18.

3. Synder C. Preocular tears film anomalies and lensrelated dryness. In: Silbert J, ed. Anterior Segment complications of Contact Lens Wear. 2nd edn. Butterworth-Heinemann;2000:3-21.

4. Bron AJ. Diagnosis of dry eye. Surv Ophthalmol (suppl 2) 2001; 45:221-26.

5. Caffery B, Richter D, Simpson T, Fonn D, Dougherty M, Gordon K. The prevalence of dry eye in CL wearers: part 2 of the Canadian epidemiology study. Invest Ophthalmol VisSci (Suppl) 1996;37:35-72.

6. Nichols JJ, Sinnott LT. Tear film, contact lens, and patient related factors associated with contact lens related dry eye. Invest Ophthalmol Vis Sci (suppl)2006;47:1319-28.

7. Guillon M. Dry eye symptomatology of soft contact lens wearers and non wearers. Optom and Vis Sci 2005;82:829-34.

8. McMonnies CW. Key questions in a dry eye history. J Am Optom Assoc 1986;57:512-17.

9. Patel S, Murray D, McKenzie A, Shearer DS, McGrath BD. Effects of fluorescein on tear break up time and on tear thinning time. Am J Optom Phy 
Opt 1985;62:188-90.

10. Guillon et al. Pre-ocular tear film characteristics of non wearers and soft contact lens wearers. Optom and Vis sci 1997;74:273-79.

11. Lemp MA, Hamill JR. Factors affecting tear film break up time in normal eyes. Arch Opthalmol 1973;89:103-5.

12. Nelson ND. Diagnosis of KCS. Int Ophthalmol Clin 1994;34:37-56.

13. Golding TR, Bruce AS, Mainstone JC. Relationship between tear meniscus parameters and tear break up time. Cornea 1997;16:649-61.

14. Nichols JJ, Sinnott LT. Tear film, contact lens and patient related factors associated with contact lens related dry eye. Invest Ophthalmol and Vis Sci 2006;47:1319-28.

15. Chopra SK, George S, Daniel R. Tear film break up time in non contact lens wearers and contact lens wearers in normal Indian population. Indian journal of Ophthalmology 1985;33:213-16.

16. Miller WL, doughty MJ, Narayanan S, Leach NE, Tran A, Gaume A, Bergmanson JPG.

Comparison of tear volume (by TMH and PRT) and tear fluid osmolarity measures in non lens wearers and in CL wearers. Eye \& contact lens: Science and clinical practice $2004 ; 30: 132-7$.

17. Glasson MJ, Stapleton F, Keay L, Sweeney D, Willcox MDP. Differences in clinical parameters and tear film of tolerant and intolerant contact lens wearers. Invest Ophthalmol and Vis Sci 2003;44:5116-24.

18. du Toit R, Situ P, Simpson T, Fonn D. The effects of six months of contact lens wear on tear film, ocular surfaces, and symptoms of presbyopes. Optom Vis Sci 2001;78:455-62.

19. Fonn D, Situ P, Simpson P. Hydrogel lens dehydration and subjective comfort and dryness ratings in symptomatic and asymptomatic CL wearers. Optom and Vis Sci 1999;76:700-4.

20. Santodomingo RJ, Wolffsohn JS, Gilmartin B.
Changes in ocular physiology, tear film characteristics, and symptomatology with 18 months silicon hydrogel CL wear. Optom and Vis Sci. 2006;83:73-81.

21. Kallarackal GU, Ansari EA, Amos N, Martin JC, Lane C, Camilleri JP. A comparative study to assess the clinical use of fluorescein meniscus time (FMT) with tear break up time (TBUT) and Schirmer's test (ST) in the diagnosis of dry eyes. Eye 2002;16:594-0. 